Review

\title{
Neurological Manifestations of Celiac Disease and Non-Celiac Gluten Sensitivity and the Effect of a Gluten Free Diet
}

\author{
Jamie Zhen, M. Ines Pinto-Sanchez*
}

Farncombe Family Digestive Health Research Institute, McMaster University, Hamilton, Canada; EMails: jamie.zhen@medportal.ca; pintosm@mcmaster.ca

* Correspondence: M. Ines Pinto-Sanchez; E-Mail: pintosm@mcmaster.ca

Academic Editor: Luis Rodrigo

Special Issue: Gluten-Related Neurological Disorders

OBM Neurobiology

2020, volume 4, issue 3

doi:10.21926/obm.neurobiol.2003065
Received: May 17, 2020

Accepted: July 08, 2020

Published: July 10, 2020

\begin{abstract}
Celiac disease (CeD) is an immune-mediated enteropathy triggered by gluten ingestion and affects nearly $1 \%$ of the population worldwide. CeD has typically been associated with gastrointestinal symptoms such as diarrhea and abdominal pain; however, its extraintestinal manifestations are becoming widely recognized. In particular, CeD patients may present with various neurological manifestations and oftentimes, without evidence of gastrointestinal symptoms. The pathophysiology behind these manifestations is not well known, however, it is hypothesized that they may include vitamin deficiencies from malabsorption, direct gluten toxicity, and immune-mediated inflammation. The most common neurological manifestations include gluten ataxia, migraines, epilepsy, and neuropathies, but may also involve dementia, myopathies, and movement disorders. The first line treatment for CeD is typically a strict gluten-free diet, however its effect on neurological manifestations is less clear. Non-celiac gluten sensitivity/non-celiac wheat sensitivity (NCGS/NCWS) represents a condition where the ingestion of gluten causes symptoms similar to CeD, but CeD-specific enteropathy or serology are both absent. Several lines of research do not differentiate CeD and NCGS/NCWS and as a result, NCGS/NCWS is often under-studied. This study seeks to review the evidence regarding the neurological
\end{abstract}

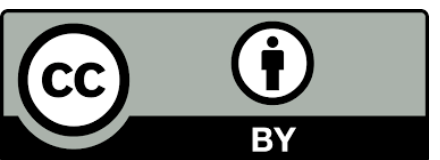

(C) 2020 by the author. This is an open access article distributed under the conditions of the Creative Commons by Attribution License, which permits unrestricted use, distribution, and reproduction in any medium or format, provided the original work is correctly cited. 
manifestations of CeD, the effect of a gluten-free diet, and highlight studies investigating NCGS/NCWS as a distinct entity from CeD.

\section{Keywords}

Gluten, celiac disease, gluten sensitivity, wheat sensitivity, neurological disorders, extraintestinal manifestations

\section{Introduction}

Celiac disease (CeD) is a chronic immune-mediated enteropathy of the small intestine that is triggered by dietary gluten intake in genetically predisposed individuals [1]. It is a relatively common disorder that affects nearly $1 \%$ of the population worldwide [2]. Studies investigating CeD have suggested a strong genetic component to its pathogenesis, as more than $95 \%$ of cases express the haplotypes HLA-DQ2 or HLA-DQ8. However, HLA-DQ2 and HLA-DQ8 haplotypes are present in up to $40 \%$ of the general population $[3,4]$. Therefore, environmental factors such as intestinal infections, alterations in the gut microbiota, and age of gluten introduction are suspected to also play a role in the development of CeD [3].

Gluten, a storage protein found in wheat, barley and rye, precipitate immune-mediated inflammatory processes within the small intestine in patients with CeD [5]. The auto antibodies, anti-tissue transglutaminase, anti-endomysium, and anti deaminated-gliadin peptides, are typically associated with CeD and used for diagnosis [6]. As a result of the inflammation, CeD generally causes villous atrophy, crypt hyperplasia, and marked infiltration of lymphoid cells into the intestinal epithelium [7]. These alterations in mucosal architecture lead to a reduction in absorptive surface area and ultimately results in malabsorption. Due to the various histological changes in CeD, small bowel biopsy is often the gold standard for diagnosis. As a consequence of malabsorption, CeD typically presents with diarrhea, weight loss, abdominal pain, and steatorrhea [8]. However, the classical picture focussing on the intestinal symptoms of CeD has been decreasing over time; whereas its extraintestinal manifestations and asymptomatic presentations have been gaining prominence [8]. The extraintestinal manifestations of CeD are often multisystemic and include hematological, mucocutaneous, musculoskeletal, psychological, gynecological, and neurological conditions [9].

Gluten can also cause symptoms in the absence of CeD. NCGS/NCWS is a condition characterized by intraintestinal or extraintestinal symptoms following the ingestion of gluten in patients without CeD or wheat allergy [10]. In NCGS/NCWS, symptoms nearly mimic those of CeD; however, in contrast to patients with CeD, patients with NCGS/NCWS do not have the autoantibodies or villous atrophy that is characteristic of CeD [11]. Due to lack of validated biomarkers, NCGS/NCWS can only be diagnosed after CeD is ruled out. In both CeD and NCGS/NCWS, the main treatment is strict life-long adherence to a gluten-free diet (GFD). Although not universal, strict adherence to a GFD typically results in the normalization of serological markers, improvement in intestinal mucosal abnormalities, and improvement of symptoms [4].

In the past few decades, the percentage of CeD and NCGS/NCWS patients that initially present atypically has been steadily increasing [12]. The uncertainty behind the extraintestinal 
manifestations are further compounded as the pathogenesis of the many associated conditions are unclear. However, it is hypothesized that some may involve vitamin and nutrient deficiencies resulting from malabsorption, while others may involve various immune-mediated pathways [13].

Notably, the neurological manifestations of CeD are one of the major domains of investigation as patients may present with neurological disorders as their first or only symptom of CeD. It has been found that as many as $49 \%$ of CeD patients present with neurological changes [9]. These disorders include peripheral neuropathy, gluten ataxia, epilepsy, headaches, cognitive deficiency, and encephalopathy [9]. The prevalence of neurological symptoms in CeD patients is estimated to be approximately $8 \%$, while a study conducted on patients with cryptogenic neurological disorders found that $57 \%$ had anti-gliadin antibodies (AGA), a non-specific antibody initially associated with CeD, and more recently to NSGS/NCWS $[14,15]$. With delays in diagnosis and ultimately left untreated, patients could develop permanent neurological disabilities such as advanced dementia and irreversible damage such as Purkinje cell loss [16]. Therefore, it is important to consider CeD and NCGS/NCWS within the differential diagnosis when investigating for neurological disorders.

We have performed a review of the literature on the evidence regarding the neurological manifestations of CeD and NCGS/NCWS, and the effect of a gluten-free diet.

\section{Materials and Methods}

We searched the Medline (PubMed) database to identify studies involving the neurological conditions associated with CeD and NCGS/NCWS. The search strategy can be found in Supplementary data. We further screened the reference lists of included studies and related systematic reviews that were previously published to identify any additional relevant studies.

Studies were included regardless of study design, geographical location, and year of publication. We included studies up to April 2020, published in English, and additionally included those that reported the effects of the implementation of a gluten-free diet (GFD) on the neurological manifestations of NCGS/NCWS. Studies investigating psychological alterations, such as anxiety disorders and mood disorders, were excluded from this review. This review is primarily concerned with the investigation, summary, and critical analysis of the neurological manifestations of CeD and NCGS/NCWS and their relationship with the implementation of a GFD.

We assessed the quality of the body of evidence using the GRADE approach (Grading of Recommendations Assessment, Development and Evaluation) [17]. GRADE specifies four levels of quality: high, moderate, low, and very low, based on five factors that ultimately decrease the quality of evidence: risk of bias, inconsistency, indirectness, imprecision, and publication bias. A summary of the main studies exploring the effects of a GFD for the neurological manifestations, and quality of evidence using GRADE approach for each neurological condition of CeD and NCGS/NCWS is shown in Table 1. 
Table 1 Main studies exploring the effects of a GFD for the neurological manifestations of CeD and NCGS/NCWS.

\begin{tabular}{|c|c|c|c|c|c|c|}
\hline Manifestation & Condition & Author (year) & Study design & Population & Outcome & GRADE score \\
\hline \multirow[t]{3}{*}{ Gluten ataxia } & CeD & $\begin{array}{l}\text { Mearns et al } \\
\text { (2019) }\end{array}$ & Systematic Review & $\begin{array}{l}9 \text { studies on gluten } \\
\text { ataxia }\end{array}$ & $\begin{array}{l}\text { Controversial findings; improvement in } \\
\text { cerebellar functioning after GFD versus no } \\
\text { improvements in self-reported ataxia } \\
\text { assessments }\end{array}$ & $\begin{array}{l}\text { Low } \\
\text { (downgraded } \\
\text { due to } \\
\text { imprecision, } \\
\text { inconsistency, } \\
\text { and risk of bias) }\end{array}$ \\
\hline & & $\begin{array}{l}\text { Hadjivassiliou } \\
\text { et al (2017) }\end{array}$ & $\begin{array}{l}\text { Prospective } \\
\text { observational } \\
\text { study }\end{array}$ & $\begin{array}{l}117 \text { CeD patients } \\
\text { with gluten ataxia }\end{array}$ & $\begin{array}{l}\text { Improvement in cerebellar } N \text {-acetylaspartate } \\
\text { /creatine levels }\end{array}$ & $N / A$ \\
\hline & NCGS/NCWS & $\begin{array}{l}\text { Hadjivassiliou } \\
\text { et al (2003) }\end{array}$ & Case-control study & $\begin{array}{l}43 \text { patients with } \\
\text { gluten ataxia }\end{array}$ & $\begin{array}{l}\text { Improvement in every ataxia test metric and } \\
\text { clinical impression }\end{array}$ & N/A \\
\hline \multirow[t]{2}{*}{$\begin{array}{l}\text { Migraines/ } \\
\text { headaches }\end{array}$} & CeD & Zis et al (2018) & $\begin{array}{l}\text { Systematic review } \\
\text { and meta-analysis }\end{array}$ & $\begin{array}{l}40 \text { studies in } \\
\text { pediatric and adult } \\
\text { CeD populations } \\
\text { with headaches }\end{array}$ & $\begin{array}{l}\text { Reduction in headache frequency in } 51.6 \% \text { - } \\
100 \% \text { of adult patients and } 69.2 \%-100 \% \text { in } \\
\text { children on a GFD }\end{array}$ & $\begin{array}{l}\text { Low } \\
\text { (downgraded } \\
\text { due to risk of } \\
\text { bias in the } \\
\text { studies and } \\
\text { large } \\
\text { inconsistencies) }\end{array}$ \\
\hline & NCGS/NCWS & $\begin{array}{l}\text { Tovoli et al } \\
\text { (2019) }\end{array}$ & $\begin{array}{l}\text { Observational } \\
\text { study }\end{array}$ & $\begin{array}{l}44 \text { patients with } \\
\text { NCWS (Salerno } \\
\text { criteria) }\end{array}$ & Decrease in headache frequency after a GFD & $N / A$ \\
\hline Epilepsy & $\begin{array}{l}\text { Mixed } \\
\text { population } \\
\text { of CeD and }\end{array}$ & $\begin{array}{l}\text { Julian et al } \\
\text { (2019) }\end{array}$ & $\begin{array}{l}\text { Systematic review } \\
\text { and meta-analysis }\end{array}$ & $\begin{array}{l}39579 \text { pooled } \\
\text { patients with } \\
\text { epilepsy, CeD, or }\end{array}$ & $\begin{array}{l}\text { Controversial; slight majority of patients in } \\
\text { the included studies were responsive to a } \\
\text { GFD ( } 53 \% \text { responsive, } 47 \% \text { non-responsive) }\end{array}$ & $\begin{array}{l}\text { Low } \\
\text { (downgraded } \\
\text { due to high risk }\end{array}$ \\
\hline
\end{tabular}


OBM Neurobiology 2020; 4(3), doi:10.21926/obm.neurobiol.2003065

NCGS/NCWS

Neuropathy
Mearns et al
(2019)

Tursi et al

(2006)

Simonati et al

(1998)

\section{Mixed}

population

of $\mathrm{CeD}$ and

NCGS/NCWS
Hadjivassiliou Case-control study

et al (2006)

Hadjivassiliou

et al (2010)

Zis et al (2017)

observational case

series

Case-control study
Systematic review

13 studies on gluten

neuropathy

12 CeD patients with

peripheral

neuropathy

1 child with CeD and

progressive

polyneuropathy

35 patients with

gluten neuropathy

17 patients with

sensory

ganglionopathy
53 patients with

gluten neuropathy
Controversial findings; improvement in neuropathic symptoms after GFD

implementation, but GFD adherence may

not prevent or reverse neuropathic damage

No differences in TNS/anti-neuronal antibodies from GFD versus baseline

Progression in neuropathy despite GFD

Improvement in sural SNAP amplitude and subjective neuropathy symptoms

Stabilization in neuropathy symptoms after GFD adherence

Improvement in pain and health change domains of SF-36 after GFD of bias in the

included studies

and

inconsistencies)

Low

(downgraded

due to

imprecision, inconsistency, and risk of bias)

N/A

N/A

N/A

N/A

N/A 


\section{Extraintestinal Manifestations - Neurological Disorders in CeD and NCGS/NCWS}

\subsection{Gluten Ataxia}

Gluten ataxia (GA) is one of the most common neurological manifestations of CeD. It was initially defined as an idiopathic sporadic ataxia in the presence of anti-gliadin antibodies [18]. The main clinical manifestation of GA is characterized by the impairment of motor abilities. Patients typically present with difficulty with limb control, gait instability, abnormalities with eye movement, speech issues, and the loss of fine motor skills $[19,20]$. Therefore, GA typically mimics the symptoms of pure cerebellar ataxia and often lacks clinical features that can distinguish the two [21].

GA frequently develops insidiously and is often not associated with the typical gastrointestinal symptoms of CeD [22]. In fact, typically less than $10 \%$ of patients with GA exhibit gastrointestinal symptoms and only a third have signs of enteropathy on biopsy. GA often develops in adulthood, affects both sexes equally, and has a mean age at onset of 53 years [23]. The prevalence of GA in $\mathrm{CeD}$ is estimated to be up to $6 \%$, and constitutes $19 \%-41 \%$ of all neurological manifestations in patients with CeD [14, 19, 24, 25].

The underlying pathogenesis of GA remains unclear; however, a few theories have been postulated. It has been suggested that the anti-gliadin antibodies, or gliadin itself, could be directly neurotoxic to the cerebellum [26]. Another hypothesis postulates that it is vitamin E deficiency or autoimmune targeting of the cerebellum that is responsible for the development of gluten ataxia $[27,28]$. The autoimmune hypothesis is supported by findings that suggest that the Purkinje cells of the cerebellum share similar epitopes with gliadin which may result in cross reactivity with antigliadin antibodies [26]. Overall, patients with GA typically have abnormalities that involve the cerebellum on magnetic resonance imaging (MRI) and magnetic resonance spectrometry [18]. These changes include cerebellar atrophy, loss of Purkinje cells, cerebellar inflammation, oligoclonal bands in the cerebrospinal fluid, and anti-Purkinje cell antibodies [29, 30]. In fact, cerebellar atrophy is found in up to $60 \%$ of patients with gluten ataxia, while nearly $100 \%$ have spectroscopic abnormalities that affect the vermis [18]. Diagnosis is typically based on clinical findings and confirmed if there is stabilization or improvement in symptoms after a GFD [31].

Early evidence behind the treatment of GA with a GFD has been controversial; especially case reports [29, 32]. However, recent studies in larger cohorts have demonstrated improvements in GA symptoms after following a GFD [33, 34]. A systematic review conducted by Mearns et al. further noted controversial findings and speculated that the differences may be due to the different methodology used to measure GFD adherence and neurological improvement [19]. Further, a study of $117 \mathrm{CeD}$ patients with GA was separated into 3 groups; strict GFD adherence (negative serology), partial adherence (presence of AGA), or declined a GFD. A baseline magnetic resonance spectroscopy scan was compared to another scan after a mean interval of 22 months and demonstrated significant improvements in cerebellar $\mathrm{N}$-acetylaspartate/creatine levels (a biomarker of neuronal functionality) after following a strict GFD [34]. Notably, the effect of a GFD was not dependent on the presence of enteropathy [33, 34].

Studies that investigate GA in patients with NCGS/NCWS is quite limited. A retrospective analysis of 334 patients with NCGS/NCWS (categorized by the authors as the presence of AGA in 
the absence of enteropathy) and 228 patients with CeD presenting with neurological dysfunction found that $46 \%$ of NCGS/NCWS patients had GA compared to $41 \%$ in the CeD group [24]. The researchers noted that GA responded to a GFD in both groups. A more recent study evaluated anti-gliadin antibodies ( $A G A$ ) in 31 patients with GA and found elevated AGA in $100 \%$ of the cases. According to the authors, this elevation in antibody levels was more frequently seen in NCGS/NCWS (classified as self-reported gluten intolerance, negative celiac serology, and the absence of villous atrophy) when compared to CeD (89 vs 48\%) [35]. Further, a case-control study involving 43 patients presenting with gluten ataxia was conducted by recording ataxia test metrics and using a subjective global clinical impression scale [33]. After 1 year of following a strict GFD, confirmed by the elimination of anti-gliadin antibodies, there was an improvement in every metric measured compared to the control group.

\subsection{Migraines/Headaches}

Migraines are a commonly reported neurological complaint in patients with CeD and NCGS/NCWS. They are characterized as a recurrent headache disorder and can often drastically affect one's quality of life. Over $20 \%$ of people worldwide suffer from migraines at some point in their lives [36]. They typically present as a moderate to severe, unilateral, throbbing headache and involve nausea, vomiting, photophobia, or phonophobia [37]. Migraines have been documented as the initial and only manifestation of CeD in numerous studies [38-40].

In a large population-based study, the number of headache-related medical visits was compared between 28,638 CeD patients and 143,126 controls [41]. They found a higher percentage of $\mathrm{CeD}$ patients (4.7\%) visited for headaches compared to the controls (2.9\%). Further, a case-control study investigated 90 patients with idiopathic migraines and found that $4.4 \%$ had CeD compared to $0.4 \%$ in the controls [42]. Overall, patients with CeD are at a higher risk of developing migraines as demonstrated by a meta-analysis conducted by Zis et al [43]. They noted that the mean prevalence of headaches in adult patients with CeD was $26 \%$ and significantly higher when compared to controls (OR 2.7, 95\% Cl 1.7-4.3, $p<0.0001$ ).

Imaging studies performed on patients with $\mathrm{CeD}$ complaining of migraines often show abnormalities. On computed tomography (CT) scans, there have been reports of occipital and parieto-occipital calcifications in patients with no evidence of epilepsy [44, 45]. On magnetic resonance imaging $(\mathrm{MRI})$, there has been studies demonstrating white matter abnormalities in CeD patients with headaches [46]. A small study investigating single photon emission computed tomography (SPECT) of the brain found that all CeD patients had abnormalities suggesting cortical hypoperfusion [42]. Overall, the pathogenesis of migraines typically seems to involve brain hypoperfusion and perivascular inflammation [43].

The implementation of a GFD appears to be an effective treatment for migraines associated with CeD. A systematic review and meta-analysis demonstrated that the percentage of adults that report a significant reduction in headache frequency after GFD introduction ranges from $51.6 \%$ to $100 \%$; and complete resolution is achieved in up to $75 \%$ [43]. Similar response rates were found in children and found that there was reduction in headache frequency and normalization of EEG findings after 6 months of a GFD [40,47]. Further, it was demonstrated that strict adherence to a GFD for a period of 6 months (demonstrated by negative IgG anti-transglutaminase and negative IgA anti-endomysial antibodies) may normalize cortical hypoperfusion [42]. While patients who 
adhere to a strict GFD (characterized by negative serology) have a lower incidence of white matter abnormalities [48].

NCGS/NCWS has been associated with recurrent headaches in a relatively low number of studies. An observational study involving 49 patients with self-reported gluten sensitivity found a greater proportion of headaches in the cases when compared to a group of 734 controls [49]. Further, various studies show that headaches seem more frequent in adults compared to children with NCGS/NCWS $[50,51]$. Meanwhile, evidence regarding the effect of a GFD in patients with NCGS/NCWS and headaches is limited. A study involving 44 patients diagnosed with NCWS (confirmed by exclusion of $\mathrm{CeD} /$ wheat allergy and gluten-related symptoms that ameliorate after a GFD of at least 6 months) found that while $63.6 \%$ had headaches at baseline, this number decreased to $40.9 \%$ at a follow up of at least 1 year after GFD implementation [52].

\subsection{Epilepsy}

In both CeD and NCGS/NCWS, patients can present with variations of epileptic seizures with and without overt brain pathologies [16]. It is estimated that one-third of cases are refractory to treatment [53]. The symptoms of epileptic seizures vary markedly, however, they are associated with motor, sensory, and visual disturbances. Examples include hallucinations, abnormal sensations, twitching, loss of consciousness, and convulsions [54].

Results from a systematic review and meta-analysis found that the prevalence of CeD amongst individuals with epilepsy was $2.27 \%$ in adults, $1.83 \%$ in children, and $3.8 \%$ in studies with serological tests for general gluten sensitivity [55]. The overall pooled prevalence of epilepsy in patients with CeD was calculated to be $1.14 \%$. These figures are consistently higher than what is found in the general population (0.64\%) and suggest that there is an increased risk of epilepsy in CeD patients [53]. Similar to the other neurological manifestations, $40 \%$ of patients with CeD and epilepsy do not report having any gastrointestinal symptoms [55]. However, in contrast to the other manifestations which typically present later in life, epilepsy more commonly develops during childhood in patients with CeD [55].

The pathophysiology behind epilepsy in CeD patients is not fully understood. However, it is hypothesized that folate deficiency, immune reactions associated with anti-gliadin antibodies, and cerebral calcifications (CEC) play a role in its pathogenesis and are commonly found in patients with CeD and epilepsy [56, 57]. There are several different presentations of epilepsy in the context of CeD with CEC being the best characterized. CEC is a neurological syndrome where patients generally have focal, pharmacologically resistant epilepsy with parieto-occipital brain calcifications detected by CT or MRI [55]. In addition, patients with epilepsy and CeD may develop transglutaminase isoenzyme 6 (TG6) autoantibodies, hippocampal sclerosis with cell loss, cortical vascular and electroencephalography (EEG) abnormalities $[58,59]$. Thus, diagnosis is typically established based on a combination of clinical, serological, EEG, and neuroimaging findings.

The implementation of a GFD could be an effective intervention to prevent epileptic seizures in patients with CeD. A systematic review of the literature demonstrated that the frequency of seizures decreased in $53 \%$ of patients with CeD following the introduction of a GFD [55]. However, cerebral calcifications associated with CEC may not resolve with the implementation of a GFD even after a follow up period of 2 years [60]. Research further suggests that the effectiveness of a GFD is inversely proportional to the duration of epilepsy prior to GFD introduction [16]. It is 
hypothesized that the effects of a GFD result from the reduction of the neurological damage caused by gluten ingestion or the resolution of its malabsorptive sequalae with subsequent increase in folate, vitamin, and anti-epileptic drug absorption [49]. Therefore, this highlights the importance of early implementation of a GFD to improve the control of epilepsy in CeD patients.

Research investigating the association between epilepsy and NCGS/NCWS is extremely limited. A study conducted by Hadjivassiliou et al found that epilepsy and CEC are very rare in NCGS/NCWS patients with neurological dysfunction (1 out of 334 patients with AGA and no enteropathy) [24]. a single case-report study documented a two-year-old patient with epilepsy and NCGS/NCWS (based on clinical suspicion after exclusion of CeD and wheat allergy) that responded to a GFD which persisted at follow up after 2 years [61]. Therefore, further studies are needed to assess the relationship between epilepsy and NCGS/NCWS.

\subsection{Neuropathy}

Peripheral neuropathy (PN), is one of the most common neurological manifestations of CeD and NCGS/NCWS. [62]. The symptoms of PN include paresthesia, numbness, pain, muscle weakness, and autonomic symptoms [63]. The prevalence of PN in patients with CeD is estimated to be up to 49\%; a risk 2.3-5.6 times greater than the general population [19,64]. However, patients often present with neuropathic symptoms even before the diagnosis of CeD is made (4). A study further found that $2.5 \%$ of patients being investigated for idiopathic neuropathy had CeD [65].

The most common type of PN implicated in $\mathrm{CeD}$ is a chronic, symmetrical, sensorimotor peripheral neuropathy that primary affects the distal regions of the body $[65,66]$. However, a wide variety of other neuropathic conditions have been reported, such as: sensory ganglionopathy, mononeuritis multiplex, pure motor neuropathy, a Guillain-Barré-like syndrome, and autonomic neuropathy $[13,65,67]$. Epidemiological studies investigating PN in CeD patients are limited, however, female sex and an increase in age is generally associated with an increased risk [19].

The pathogenesis of PN in the context of CeD has initially been thought to be as a result of vitamin deficiencies, potentially $B_{1}, B_{6}, B_{12}$, and $E$, and inflammation by direct gluten toxicity [13, 20]. However, recent reports suggest these factors may not play a significant role and it is more likely due to perivascular infiltration of immune cells that causes neurological damage [68]. Electrophysiological studies can be normal or only mildly abnormal in CeD patients with PN [69]. Skin and nerve biopsies have been found loss of myelinated fibers, axonal degeneration, and decreases in epidermal nerve density [68]. A study of 20 patients with CeD and PN found antiganglioside antibodies in $65 \%$ of cases [70]. These auto-antibodies may bind to Schwann cells, nodes of Ranvier, and peripheral nerve axons which may ultimately contribute to PN. On physical exam, the predominant manifestation of PN is a sensory neuropathy with variable involvement of large and small fibers [65]. Therefore, after the exclusion of other etiologies, diagnosis of PN may be established with a combination of diagnostic tests and clinical findings.

The effects of a GFD in CeD patients with PN is controversial. A systematic review conducted by Mearns et al. noted that although GFD implementation may improve neuropathic symptoms, it may not prevent or reverse neuropathic damage [19]. Further, a prospective study involving 35 CeD patients on a GFD found improvements in both sural sensory nerve action potential (SNAP) amplitude and in the patients' subjective assessment of their neuropathic symptoms [71]. 
Meanwhile, a case-control study of $53 \mathrm{CeD}$ patients with neuropathy showed improvements in their pain and quality of life scores after strict GFD adherence (measured after negative celiac serology) [72]. Similar results have been noted in a retrospective study of $328 \mathrm{CeD}$ patients followed for 2 years [73]. On the contrary, a small study found no significant differences in total neuropathy score (TNS) following 1 year of GFD implementation when compared to the baseline [74]. A study involving 10 patients without enteropathy also showed stabilization of PN symptoms after 6 months of strict GFD adherence; suggesting that the effect of gluten in PN may not be limited to the CeD population [75].

In a group of 334 patients with NCGS/NCWS (characterized as positive AGA and absence of enteropathy) and neurological dysfunction, the most common neurological manifestation found was peripheral neuropathy (54\%) [24]. The researchers further noted that patients with CeD may have more severe PN when compared to NCGS/NCWS [24]. However, the vast majority of studies investigating neuropathy do not discriminate NCGS/NCWS from CeD. As a result, evidence supporting the association between PN and NCGS/NCWS is very limited. Further prospective studies in the NCGS/NCWS population are needed to determine the association with PN.

\subsection{Others}

Numerous studies suggest that there is an association between $\mathrm{CeD}$ and cognitive impairment $[76,77]$. Oftentimes, patients may present with confusion, amnesia, attention difficulties, difficulties concentrating, and reduced mental acuity [78]. Several mechanisms for the pathogenesis of cognitive impairment in CeD patients have been postulated; including nutrient deficiencies, elevation in circulating cytokine levels as a result of systemic inflammation, and the direct effect of gluten on serotonin levels [79]. Transcranial magnetic stimulation (TMS) tests of the brain typically show hyper-excitability and impaired central motor conductivity [80]. Further imaging studies of patients with cognitive impairment associated with CeD may show brain atrophy, nonspecific gliosis, and nonspecific slowing on EEG [76]. The evidence regarding the effect of a GFD for cognitive impairment in CeD is mixed. Some studies suggest that cognition improves after GFD [76, 81] while others see the opposite effect [77, 82]. However, due to its potentially protective effect, authors typically suggest that a GFD should be introduced early.

Myopathies such as inclusion body myositis, polymyositis, and dermatomyositis are neuromuscular disorders that have been associated with $\mathrm{CeD}$ [83]. Studies suggest that the risk of an idiopathic inflammatory myopathy in CeD patients may be increased 9-fold when compared to the general population [83]. Like many other manifestations of $\mathrm{CeD}$, it is speculated that myopathies may be related to an immune-mediated mechanism or vitamin deficiencies such as vitamin D or E [16]. Muscle biopsies in these patients may show muscle fiber necrosis, basophilic rimmed vacuoles, and inflammatory cell infiltrates [68]. Evidence generally suggests that clinical improvement could be attained with the implementation of a GFD for gluten-related myopathies [83-86].

A systematic review demonstrated that movement disorders such as chorea, dystonia, myoclonus, stiff-person syndrome, myokymia, paroxysmal dyskinesia, and myokymia are frequently reported in CeD [87]. The most common movement disorder noted was restless leg syndrome, possibly as consequence of iron deficiency, a finding that is commonly implicated in 
CeD [87]. Studies suggest that restless leg syndrome symptoms typically improve with a GFD, with and without iron supplementation $[88,89]$.

In Figure 1 we summarized the prevalence and pathogenesis of the most common neurological conditions in CeD and NCGS/NCWS. Table 2 shows a summary of diagnostic methods and the effect of the gluten free diet on the most common neurological conditions in $\mathrm{CeD}$ and NCGS/NCWS.

Gluten Ataxia

- 6\% of CD patients

- Cerebellar atrophy

Migraines/headaches

- 26\% of CD patients

- Cortical hypoperfusion

\section{Epilepsy}

- $1 \%$ of $C D$ patients

- Cerebral calcification

\section{Neuropathy}

- 49\% of CD patients

- Peripheral nerve perivascular inflammation

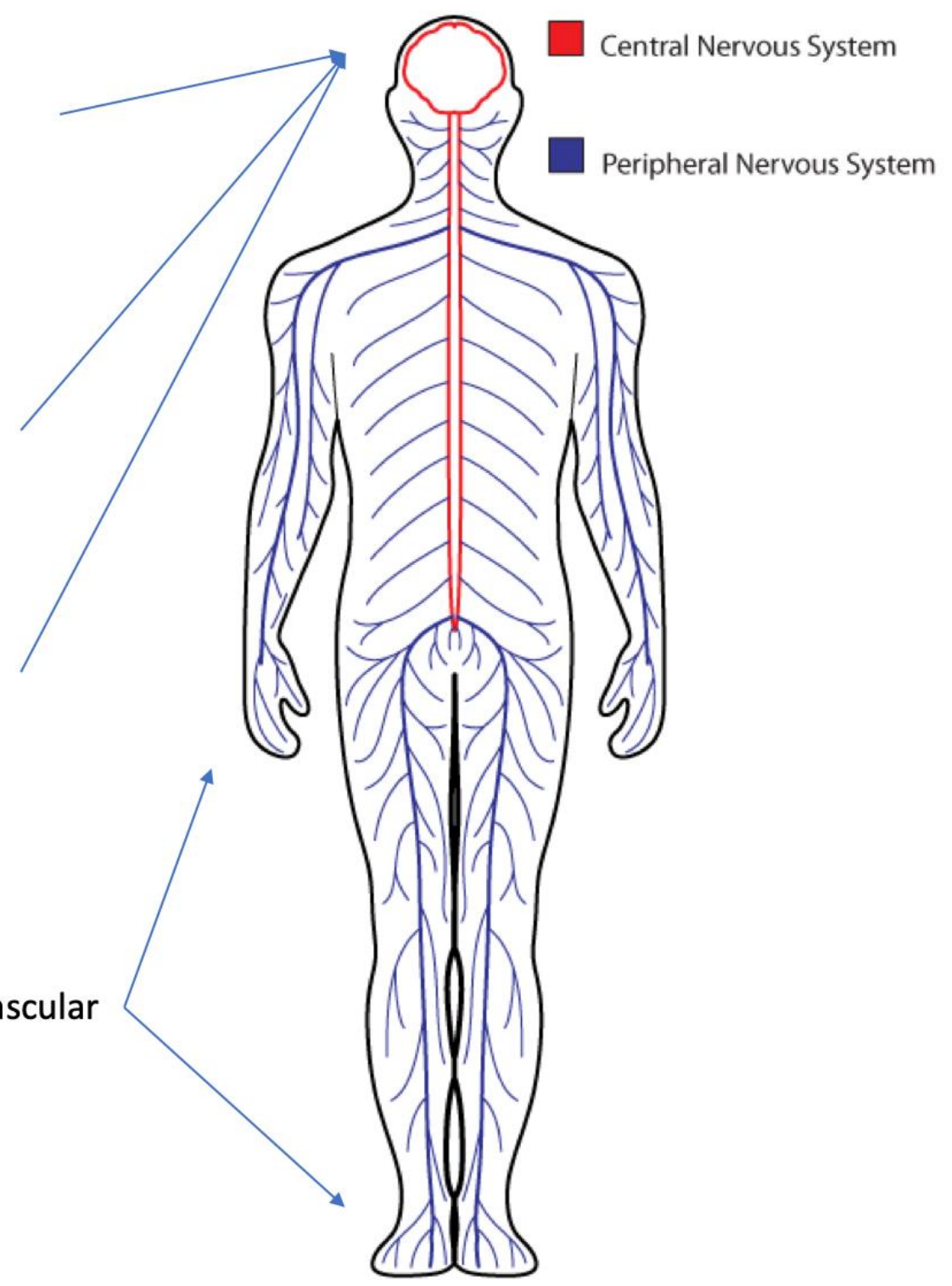

Figure 1 Prevalence and pathogenesis of neurological manifestations in CeD and NCGS/NCWS. 
Table 2 Summary of diagnostic methods and the effect of gluten free diet in the most common neurological manifestations of celiac disease and NCGS/NCWS.

\begin{tabular}{|c|c|c|c|c|}
\hline $\begin{array}{l}\text { Neurological } \\
\text { Manifestation }\end{array}$ & $\begin{array}{l}\text { Prevalence in } \\
\text { CeD }\end{array}$ & $\begin{array}{l}\text { Prevalence in } \\
\text { NCGS/NCWS }\end{array}$ & Diagnosis & $\begin{array}{l}\text { Effect of a } \\
\text { gluten-free diet }\end{array}$ \\
\hline Gluten ataxia & Up to $6 \%$ [19] & N/A & $\begin{array}{l}\text { MRI, magnetic } \\
\text { resonance } \\
\text { spectrometry } \\
{[18]}\end{array}$ & $\begin{array}{l}\text { Controversial; } \\
\text { In favour } \\
\text { [32-34] } \\
\text { Not helpful [29] }\end{array}$ \\
\hline Migraines/headaches & $26 \%[43]$ & $\begin{array}{l}12.2 \%-63.6 \% \\
{[49-52]}\end{array}$ & $\begin{array}{l}\text { CT }[44,45] \\
\text { MRI }[46] \\
\text { SPECT }[42]\end{array}$ & $\begin{array}{l}\text { In favour } \\
{[40,42,43,47 \text {, }} \\
48]\end{array}$ \\
\hline Epilepsy & $\begin{array}{l}\text { Pooled mean of } \\
1.14 \%[55]\end{array}$ & $\begin{array}{l}\text { Pooled mean of } \\
0.93 \%[55]\end{array}$ & $\begin{array}{l}\text { CT, MRI }[55] \\
\text { EEG and TG6 } \\
\text { serology }[58,59]\end{array}$ & $\begin{array}{l}\text { In favour [55] } \\
\text { Early } \\
\text { intervention } \\
\text { preferred [16] }\end{array}$ \\
\hline Neuropathy & $\begin{array}{l}\text { Up to } 49 \% \\
{[19,64]}\end{array}$ & $\begin{array}{l}54 \% \text { of } \\
\text { NCGS/NCWS } \\
\text { patients } \\
\text { presenting with } \\
\text { a neurological } \\
\text { complaint [24] }\end{array}$ & $\begin{array}{l}\text { Electrophysiology } \\
\text { studies [69] } \\
\text { Skin and nerve } \\
\text { biopsy [68] } \\
\text { Anti-ganglioside } \\
\text { serology [70] }\end{array}$ & $\begin{array}{l}\text { Controversial; } \\
\text { In favour [71-73, } \\
75] \\
\text { Not helpful } \\
\text { [74] }\end{array}$ \\
\hline
\end{tabular}

Abbreviations: N/A = Not applicable; MRI = Magnetic resonance imaging; $\mathrm{CT}=$ Computerized tomography; $\mathrm{SPECT}=$ Single photon emission computed tomography; $\mathrm{EEG}=$ Electroencephalogram; TG6 = Anti-tissue transglutaminase IgA 6; NCGS/NCWS= Non-celiac gluten sensitivity/non-celiac wheat sensitivity

\section{Discussion}

This review highlights the literature surrounding the prevalence, pathophysiology, diagnostic findings, and the effect of a GFD on the neurological manifestations of CeD and NCGS/NCWS. Peripheral neuropathy and migraines/headaches are generally known as the most common manifestations, followed by gluten ataxia and epilepsy. In all cases, the implementation of a GFD is typically recommended due to its low risk profile and potential benefits.

In addition to the neurological manifestations of CeD and NCGS/NCWS, a wide range of psychiatric symptoms and disorders including anxiety, mood disorders, and attention deficit hyperactivity disorder (ADHD) have also been found to be associated with gluten-related disorders [90]. The pathogenesis of these disorders are not fully understood, however, cerebral hypoperfusion, vitamin deficiencies, or neuro-inflammation could play a role [90]. Further, untreated $\mathrm{CeD}$ patients have been found to have neurotransmitter abnormalities including decreased plasma levels of tryptophan and monoamine precursors and decreased cerebrospinal fluid levels of serotonin and dopamine $[90,91]$. Although the mechanisms of both the neurological 
and psychiatric manifestations of gluten-related disorders are unclear, it could be speculated that the pathogenesis may follow similar mechanisms and should be further investigated.

Unfortunately, the overall quality of evidence for the studies reported are poor due to their observational nature. As a result, there are several limitations to this review. First, observational studies commonly involve selection bias during patient recruitment and publication bias as positive results are more likely to be published versus negative results. Further, many of the studies involved a relatively small number of patients which may make it difficult to estimate the true effect of GFD implementation. This limitation is further exacerbated by the clinical and methodological heterogeneities present in the reviewed studies. These include different definitions for the diagnosis of NCGS/NCWS, differences in follow up intervals (short intervals may miss neurological events such as a migraine) and differences in the definitions for the strictness of GFD adherence.

\section{Conclusion}

The classical picture of gastrointestinal symptoms has been fading in CeD and NCGS/NCWS, while the extra-intestinal manifestations are now well established. In particular, neurological manifestations have been increasing in prevalence and may often be the initial manifestation of CeD and NCGS/NCWS. Therefore, it may be beneficial to investigate for gluten-related disorders when a patient presents with a neurological condition of unknown etiology.

The treatment of gluten-related neurological manifestations with a strict life-long GFD immediately following diagnosis is generally recommended. However, the controversial findings suggest that further research is required to confirm the effects of a GFD for specific manifestations.

The vast majority of research on the neurological manifestations of gluten-related disorders refer primarily to $\mathrm{CeD}$ and are often confused with gluten sensitivity. Therefore, future large prospective studies with rigorous design methodology are needed to further investigate the association between both CeD, and particularly NCGS, and their neurological manifestations.

\section{Supplementary Data}

Medline (PubMed) Search Strategy

\#1 (celiac* OR coeliac* OR celiac disease [MeSH]

\#2 (non-celiac gluten sensitivity OR gluten sensitivity OR non-coeliac gluten sensitivity OR noncoeliac gluten sensitivity OR nonceliac gluten sensitivity)

\#3 (\#1 OR \#2)

\#4 (neurologic OR neurological OR neurological disorder* OR neurological manifestation* OR neurological complications)

\#5 (gluten ataxia OR migraine OR headache OR epilepsy OR neuropathy OR cognitive disorder OR cognition)

\#6 (\#4 OR \#5)

\#7 (gluten free OR gluten-free OR gluten-free diet OR gluten free diet OR GFD)

\#8 (\#3 AND \#6 AND \#7)

\#9 (\#8 AND Filters: Clinical Trial; Comparative Study; Controlled Clinical Trial; Meta-Analysis; Observational Study; Randomized Controlled Trial; Review; Systematic Reviews; Publication date from 1995 - 2020; Humans; English 
Yield $n=90$

\section{Author Contributions}

MIPS conceptualized the study and reviewed the manuscript. JZ performed the literature review and wrote the manuscript. Both authors approved the final version of the manuscript.

\section{Funding}

The authors did not receive any funding for this work.

\section{Competing Interests}

The authors declare that they have no competing interests.

\section{References}

1. Ludvigsson JF, Leffler DA, Bai JC, Biagi F, Fasano A, Green PH, et al. The Oslo definitions for coeliac disease and related terms. Gut. 2013; 62: 43-52.

2. Lebwohl B, Ludvigsson JF, Green PH. Celiac disease and non-celiac gluten sensitivity. BMJ. 2015; 351: h4347.

3. Lionetti $E$, Catassi $C$. The role of environmental factors in the development of celiac disease: What is new? Diseases. 2015; 3: 282-293.

4. Chin RL, Latov N, Green PH, Brannagan III TH, Alaedini A, Sander HW. Neurologic complications of celiac disease. J Clin Neuromuscul Dis. 2004; 5: 129-137.

5. Biesiekierski JR. What is gluten? J Gastroenterol Hepatol. 2017; 32: 78-81.

6. Rashid M, Lee J. Serologic testing in celiac disease: Practical guide for clinicians. Can Fam Physician. 2016; 62: 38-43.

7. Sollid LM. Coeliac disease: Dissecting a complex inflammatory disorder. Nat Rev Immunol. 2002; 2: 647-655.

8. Ludvigsson JF, Bai JC, Biagi F, Card TR, Ciacci C, Ciclitira PJ, et al. Diagnosis and management of adult coeliac disease: Guidelines from the British Society of Gastroenterology. Gut. 2014; 63: 1210-1228.

9. Pinto-Sánchez MI, Bercik P, Verdu EF, Bai JC. Extraintestinal manifestations of celiac disease. Dig Dis. 2015; 33: 147-154.

10. Catassi C, Elli L, Bonaz B, Bouma G, Carroccio A, Castillejo G, et al. Diagnosis of non-celiac gluten sensitivity (NCGS): The Salerno experts' criteria. Nutrients. 2015; 7: 4966-4977.

11. Leonard MM, Sapone A, Catassi C, Fasano A. Celiac disease and nonceliac gluten sensitivity: A review. JAMA. 2017; 318: 647-656.

12. Rampertab SD, Pooran N, Brar $\mathrm{P}$, Singh $\mathrm{P}$, Green $\mathrm{PH}$. Trends in the presentation of celiac disease. Am J Med. 2006; 119: 355.e9-355.e14.

13. Freeman HJ. Neurological disorders in adult celiac disease. Can J Gastroenterol. 2008; 22: 909911.

14. Cooke WT, Smith WT. Neurological disorders associated with adult coeliac disease. Brain. 1966; 89: 683-722. 
15. Hadjivassiliou M, Gibson A, Davies-Jones GA, Lobo AJ, Stephenson TJ, Milford-Ward A. Does cryptic gluten sensitivity play a part in neurological illness? Lancet. 1996; 347: 369-371.

16. Zis $P$, Hadjivassiliou M. Treatment of neurological manifestations of gluten sensitivity and coeliac disease. Curr Treat Options Neurol. 2019; 21: 10.

17. Atkins $D$, Best D, Briss PA, Eccles $M$, Falck-Ytter $Y$, Flottorp $S$, et al. Grading quality of evidence and strength of recommendations. BMJ. 2004; 328: 1490.

18. Hadjivassiliou M, Sanders DD, Aeschlimann DP. Gluten-related disorders: gluten ataxia. Dig Dis. 2015; 33: 264-268.

19. Mearns ES, Taylor A, Thomas Craig KJ, Puglielli S, Cichewicz AB, Leffler DA, et al. Neurological manifestations of neuropathy and ataxia in celiac disease: A systematic review. Nutrients. 2019; 11: 380.

20. Nikpour S. Neurological manifestations, diagnosis, and treatment of celiac disease: A comprehensive review. Iran J Neurol. 2012; 11: 59-64.

21. Luostarinen LK, Collin PO, Peraaho MJ, Maki MJ, Pirttila TA. Coeliac disease in patients with cerebellar ataxia of unknown origin. Ann Med. 2001; 33: 445-449.

22. Fasano A. Celiac disease: how to handle a clinical chameleon. N Engl J Med. 2003; 348: 25682570.

23. Hadjivassiliou M, Sanders DS, Grünewald RA, Woodroofe N, Boscolo S, Aeschlimann D. Gluten sensitivity: From gut to brain. Lancet Neurol. 2010; 9: 318-330.

24. Hadjivassiliou M, Rao DG, Grinewald RA, Aeschlimann DP, Sarrigiannis PG, Hoggard N, et al. Neurological dysfunction in coeliac disease and non-coeliac gluten sensitivity. Am J Gastroenterol. 2016; 111: 561-567.

25. Bürk K, Farecki ML, Lamprecht $G$, Roth $G$, Decker $P$, Weller $M$, et al. Neurological symptoms in patients with biopsy proven celiac disease. Mov Disord. 2009; 24: 2358-2362.

26. Hadjivassiliou M, Boscolo SA, Davies-Jones GA, Grünewald RA, Not T, Sanders DS, et al. The humoral response in the pathogenesis of gluten ataxia. Neurology. 2002; 58: 1221-1226.

27. Jackson CE, Amato AA, Barohn RJ. Isolated vitamin E deficiency. Muscle Nerve. 1996; 19 : 1161-1165.

28. Hadjivassiliou M, Mäki M, Sanders DS, Williamson CA, Grünewald RA, Woodroofe NM, et al. Autoantibody targeting of brain and intestinal transglutaminase in gluten ataxia. Neurology. 2006; 66: 373-377.

29. Bhatia KP, Brown P, Gregory R, Lennox GG, Manji H, Thompson PD, et al. Progressive myoclonic ataxia associated with coeliac disease. The myoclonus is of cortical origin, but the pathology is in the cerebellum. Brain. 1995; 118: 1087-1093.

30. Hadjivassiliou M, Williamson CA, Woodroofe N. The immunology of gluten sensitivity: Beyond the gut. Trends Immunol. 2004; 25: 578-582.

31. Khwaja GA, Bohra V, Duggal A, Ghuge VV, Chaudhary N. Gluten sensitivity - a potentially reversible cause of progressive cerebellar ataxia and myoclonus - a case report. J Clin Diagn Res. 2015; 9: OD07-OD08.

32. Pellecchia MT, Scala R, Perretti A, De Michele G, Santoro L, Filla A, et al. Cerebellar ataxia associated with subclinical celiac disease responding to gluten-free diet. Neurology. 1999; 53: 1606-1608.

33. Hadjivassiliou M, Davies-Jones GA, Sanders DS, Grünewald RA. Dietary treatment of gluten ataxia. J Neurol Neurosurg Psychiatry. 2003; 74: 1221-1224. 
34. Hadjivassiliou M, Grünewald RA, Sanders DS, Shanmugarajah P, Hoggard N. Effect of glutenfree diet on cerebellar MR spectroscopy in gluten ataxia. Neurology. 2017; 89: 705-709.

35. Rodrigo L, Hernandez-Lahoz C, Lauret E, Rodriguez-Peláez M, Soucek M, Ciccocioppo R, et al. Gluten ataxia is better classified as non-celiac gluten sensitivity than as celiac disease: $A$ comparative clinical study. Immunol Res. 2016; 64: 558-564.

36. Welch KM, Goadsby PJ. Chronic daily headache: Nosology and pathophysiology. Curr Opin Neurol. 2002; 15: 287-295.

37. Burstein R, Noseda R, Borsook D. Migraine: Multiple processes, complex pathophysiology. J Neurosci. 2015; 35: 6619-6629.

38. Serratrice J, Disdier P, De Roux C, Christides C, Weiller PJ. Migraine and coeliac disease. Headache. 1998; 38: 627-628.

39. Battistella PA, Mattesi P, Casara GL, Carollo C, Condini A, Allegri F, et al. Bilateral cerebral occipital calcifications and migraine-like headache. Cephalalgia. 1987; 7: 125-129.

40. Diaconu G, Burlea M, Grigore I, Anton DT, Trandafir LM. Celiac disease with neurologic manifestations in children. Rev Med Chir Soc Med Nat lasi. 2013; 117: 88-94.

41. Lebwohl B, Roy A, Alaedini A, Green PH, Ludvigsson JF. Risk of headache-related healthcare visits in patients with celiac disease: A population-based observational study. Headache. 2016; 56: 849-858.

42. Gabrielli M, Cremonini F, Fiore G, Addolorato G, Padalino C, Candelli M, et al. Association between migraine and celiac disease: Results from a preliminary case-control and therapeutic study. Am J Gastroenterol. 2003; 98: 625-629.

43. Zis $P$, Julian $T$, Hadjivassiliou $M$. Headache associated with coeliac disease: A systematic review and meta-analysis. Nutrients. 2018; 10: 1445.

44. Crosato F, Senter S. Cerebral occipital calcifications in celiac disease. Neuropediatrics. 1992; 23: 214-217.

45. D'Amico D, Rigamonti A, Spina L, Bianchi-Marzoli S, Vecchi M, Bussone G. Migraine, celiac disease, and cerebral calcifications: A new case. Headache. 2005; 45: 1263-1267.

46. Hadjivassiliou M, Grünewald RA, Lawden M, Davies-Jones GA, Powell T, Smith CM. Headache and CNS white matter abnormalities associated with gluten sensitivity. Neurology. 2001; 56: 385-388.

47. Parisi P, Pietropaoli N, Ferretti A, Nenna R, Mastrogiorgio G, Del Pozzo M, et al. Role of the gluten-free diet on neurological-EEG findings and sleep disordered breathing in children with celiac disease. Seizure. 2015; 25: 181-183.

48. Currie S, Hadjivassiliou M, Clark MJ, Sanders DS, Wilkinson ID, Griffiths PD, et al. Should we be 'nervous' about coeliac disease? Brain abnormalities in patients with coeliac disease referred for neurological opinion. J Neurol Neurosurg Psychiatry. 2012; 83: 1216-1221.

49. Van Gils T, Nijeboer P, IJssennagger CE, Sanders DS, Mulder CJ, Bouma G. Prevalence and characterization of self-reported gluten sensitivity in The Netherlands. Nutrients. 2016; 8: 714.

50. Volta U, Tovoli F, Cicola R, Parisi C, Fabbri A, Piscaglia M, et al. Serological tests in gluten sensitivity (nonceliac gluten intolerance). J Clin Gastroenterol. 2012; 46: 680-685.

51. Volta U, Bardella MT, Calabro A, Troncone R, Corazza GR. An Italian prospective multicenter survey on patients suspected of having non-celiac gluten sensitivity. BMC Med. 2014; 12: 85.

52. Tovoli F, Granito A, Negrini G, Guidetti E, Faggiano C, Bolondi L. Long term effects of glutenfree diet in non-celiac wheat sensitivity. Clin Nutr. 2019; 38: 357-363. 
53. Fiest KM, Sauro KM, Wiebe S, Patten SB, Kwon CS, Dykeman J, et al. Prevalence and incidence of epilepsy: A systematic review and meta- analysis of international studies. Neurology. 2017; 88: 296-303.

54. Stafstrom CE, Carmant L. Seizures and epilepsy: An overview for neuroscientists. Cold Spring Harb Perspect Med. 2015; 5: a022426.

55. Julian T, Hadjivassiliou M, Zis P. Gluten sensitivity and epilepsy: A systematic review. J Neurol. 2019; 266: 1557-1565.

56. Gobbi G. Coeliac disease, epilepsy and cerebral calcifications. Brain Dev. 2005; 27: 189-200.

57. Visakorpi JK, Kuitunen P, Pelkonen P. Intestinal malabsorption: A clinical study of 22 children over 2 years of age. Acta Paediatr Scand. 1970; 59: 273-280.

58. Struck AF, Beinlich BR, Rutecki PA. A case of celiac disease, epilepsy, and cerebral calcifications with temporal lobe epilepsy. WMJ. 2015; 114: 116-117.

59. Bye AM, Andermann F, Robitaille $\mathrm{Y}$, Oliver M, Bohane $T$, Andermann E. Cortical vascular abnormalities in the syndrome of celiac disease, epilepsy, bilateral occipital calcifications, and folate deficiency. Ann Neurol. 1993; 34: 399-403.

60. Arroyo HA, De Rosa S, Ruggieri V, De Davila MT, Fejerman N. Epilepsy, occipital calcifications, and oligosymptomatic celiac disease in childhood. J Child Neurol. 2002; 17: 800-806.

61. Bruni O, Dosi C, Luchetti A, Della Corte M, Riccioni A, Battaglia D, et al. An unusual case of drug-resistant epilepsy in a child with non-celiac gluten sensitivity. Seizure. 2014; 23: 674-676.

62. Zis P, Rao DG, Sarrigiannis PG, Aeschlimann P, Aeschlimann DP, Sanders D, et al. Transglutaminase 6 antibodies in gluten neuropathy. Dig Liver Dis. 2017; 49: 1196-1200.

63. Zis $P$, Sarrigiannis PG, Rao DG, Hewamadduma C, Hadjivassiliou M. Chronic idiopathic axonal polyneuropathy: A systematic review. J Neurol. 2016; 263: 1903-1910.

64. Cicarelli G, Della Rocca G, Amboni M, Ciacci C, Mazzacca G, Filla A, et al. Clinical and neurological abnormalities in adult celiac disease. Neurol Sci. 2003; 24: 311-317.

65. Chin RL, Sander HW, Brannagan TH, Green PH, Hays AP, Alaedini A, et al. Celiac neuropathy. Neurology. 2003; 60: 1581-1585.

66. Hadjivassiliou M, Sanders DS, Grünewald RA, Woodroofe N, Boscolo S, Aeschlimann D. Gluten sensitivity: from gut to brain. Lancet Neurol. 2010; 9: 318-330.

67. Zis P, Hadjivassiliou M, Sarrigiannis PG, Barker AS, Rao DG. Rapid neurophysiological screening for sensory ganglionopathy: A novel approach. Brain Behav. 2017; 7: e00880.

68. Rouvroye MD, Zis P, Van Dam AM, Rozemuller AJ, Bouma G, Hadjivassiliou M. The neuropathology of gluten-related neurological disorders: A systematic review. Nutrients. 2020; 12: 822.

69. Bushara KO. Neurologic presentation of celiac disease. Gastroenterology. 2005; 128: S92-S97.

70. Alaedini A, Wirguin I, Latov N. Ganglioside agglutination immunoassay for rapid detection of autoantibodies in immune-mediated neuropathy. J Clin Lab Anal. 2001; 15: 96-99.

71. Hadjivassiliou M, Kandler RH, Chattopadhyay AK, Davies-Jones AG, Jarratt JA, Sanders DS, et al. Dietary treatment of gluten neuropathy. Muscle Nerve. 2006; 34: 762-766.

72. Zis $P$, Sarrigiannis PG, Rao DG, Hadjivassiliou M. Quality of life in patients with gluten neuropathy: A case-controlled study. Nutrients. 2018; 10: 662.

73. Jericho H, Sansotta N, Guandalini S. Extraintestinal manifestations of celiac disease: Effectiveness of the gluten-free diet. J Pediatr Gastroenterol Nutr. 2017; 65: 75-79. 
74. Tursi A, Giorgetti GM, lani C, Arciprete F, Brandimarte G, Capria A, et al. Peripheral neurological disturbances, autonomic dysfunction, and antineuronal antibodies in adult celiac disease before and after a gluten-free diet. Dig Dis Sci. 2006; 51: 1869-1874.

75. Hadjivassiliou M, Rao DG, Wharton SB, Sanders DS, Grünewald RA, Davies-Jones AG. Sensory ganglionopathy due to gluten sensitivity. Neurology. 2010; 75: 1003-1008.

76. Hu WT, Murray JA, Greenaway MC, Parisi JE, Josephs KA. Cognitive impairment and celiac disease. Arch Neurol. 2006; 63: 1440-1446.

77. Collin P, Pirttila T, Nurmikko T, Somer H, Erila T, Keyrilainen O. Celiac disease, brain atrophy, and dementia. Neurology. 1991; 41: 372.

78. Lurie Y, Landau DA, Pfeffer J, Oren R. Celiac disease diagnosed in the elderly. J Clin Gastroenterol. 2008; 42: 59-61.

79. Makhlouf S, Messelmani M, Zaouali J, Mrissa R. Cognitive impairment in celiac disease and non-celiac gluten sensitivity: Review of literature on the main cognitive impairments, the imaging and the effect of gluten free diet. Acta Neurol Belg. 2018; 118: 21-27.

80. Lanza G, Bella R, Cantone M, Pennisi G, Ferri R, Pennisi M. Cognitive impairment and celiac disease: Is transcranial magnetic stimulation a trait d'Union between Gut and Brain? Int J Mol Sci. 2018; 19: 2243.

81. Lichtwark IT, Newnham ED, Robinson SR, Shepherd SJ, Hosking P, Gibson PR, et al. Cognitive impairment in coeliac disease improves on a gluten-free diet and correlates with histological and serological indices of disease severity. Aliment Pharmacol Ther. 2014; 40: 160-170.

82. Casella S, Zanini B, Lanzarotto F, Ricci C, Marengoni A, Romanelli G, et al. Cognitive performance is impaired in coeliac patients on gluten free diet: A case-control study in patients older than 65 years of age. Dig Liver Dis. 2012; 44: 729-735.

83. Danielsson O, Lindvall B, Hallert C, Vrethem M, Dahle C. Increased prevalence of celiac disease in idiopathic inflammatory myopathies. Brain Behav. 2017; 7: e00803.

84. Kleopa KA, Kyriacou K, Zamba-Papanicolaou E, Kyriakides T. Reversible inflammatory and vacuolar myopathy with vitamin E deficiency in celiac disease. Muscle Nerve. 2005; 31: 260265.

85. Selva-O'Callaghan A, Casellas F, De Torres I, Palou E, Grau-Junyent JM, Vilardell-Tarres M. Celiac disease and antibodies associated with celiac disease in patients with inflammatory myopathy. Muscle Nerve. 2007; 35: 49-54.

86. Hardoff D, Sharf B, Berger A. Myopathy as a presentation of coeliac disease. Dev Med Child Neurol. 1980; 22: 781-783.

87. Vinagre-Aragon A, Zis P, Grünewald RA, Hadjivassiliou M. Movement disorders related to gluten sensitivity: A systematic review. Nutrients. 2018; 10: 1034.

88. Manchanda S, Davies CR, Picchietti D. Celiac disease as a possible cause for low serum ferritin in patients with restless legs syndrome. Sleep Med. 2009; 10: 763-765.

89. Weinstock LB, Walters AS, Mullin GE, Duntley SP. Celiac disease is associated with restless legs syndrome. Dig Dis Sci. 2010; 55: 1667-1673.

90. Zingone F, Swift GL, Card TR, Sanders DS, Ludvigsson JF, Bai JC. Psychological morbidity of celiac disease: A review of the literature. United European Gastroenterol J. 2015; 3: 136-145.

91. Urban-Kowalczyk M, CEmigielski J, Gmitrowicz A. Neuropsychiatric symptoms and celiac disease. Neuropsychiatr Dis Treat. 2014; 10: 1961-1964. 


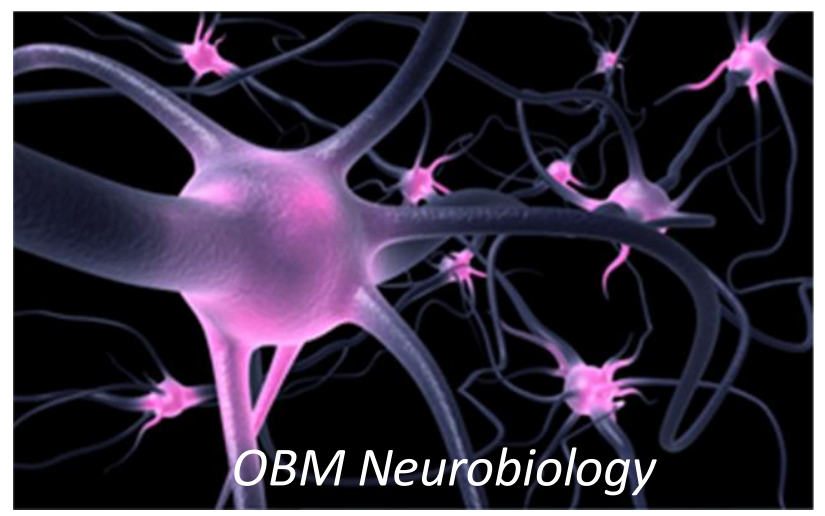

Enjoy OBM Neurobiology by:

1. Submitting a manuscript

2. Joining volunteer reviewer bank

3. Joining Editorial Board

4. Guest editing a special issue

For more details, please visit:

http://www.lidsen.com/journals/neurobiology 